\title{
Model for determining vehicle flow at roundabout sections
}

\author{
Mustafa Mehanović \\ University of Sarajevo, Faculty of Traffic and Communications, Zmaja od Bosne br. 8, Sarajevo 71000, Bosnia and Herzegovina
}

\begin{abstract}
This paper presents a model push on which to determine the flow rates vehicles per share on the basis of circular intersections recorded entries and exits of vehicles in the aisles. In addition, it is possible to analyze the influence of individual flows at intersections capacity, and determine the number of vehicles at the intersection of routes in the knowledge of the number of vehicles leaving the intersection at the next exit. Count the number of vehicles it is easier to manually and by using new technologies. Model results in a longer or shorter period of time which gives both static and dynamic characteristics.
\end{abstract}

Keywords: Intersection roundabout, Traffic flow, Traffic intersections, Model, Capacity intersections

\section{Introduction}

Research vehicle flows at roundabouts by counting vehicles by direction is very difficult. Recording of vehicle flows in intersections of roads that intersect at an angle, intersections "T" and "Y" type, the model of counting vehicles by direction can be applied because at the entrance to the intersection or on a short road at the intersection, it is possible to conclude in which direction the vehicle will get out of the intersection.

At roundabouts, especially with a larger number of approaches, with a larger radius, or with more than one traffic lane, the vehicle would have to be followed from a certain approach to the exit in order to be attributed to a certain direction of turn. For an intersection of 4 approaches, it is necessary to have 12 counters at the same time, and each counter must monitor each vehicle from the entrance to the exit, which is not possible with increased flow. In addition, the counter should take into account the structure of the vehicle, i.e. which group the vehicle belongs to, which further burdens it [1].

The application of intelligent technologies for recording currents in directions at roundabouts is also, despite the high cost, difficult and the question of the reliability of the results can be questioned.

The HCM 2000 methodology presents procedures for the analysis of roundabouts with input data on vehicle movement in directions, which makes it difficult to apply to intersections with a larger number of approaches.
The proposed Model for the Research of Roundabout Flows (abbreviated MRRF) is based on counting vehicle entrances and exits at access routes, without counting vehicles by directions. The proposed model describes the method of determining the flow of vehicles per section within intersections and vehicle flows by turning directions. Counting through counters is facilitated, and the application of new technologies at reasonable costs gives very good results.

\section{Basic elements of a roundabout intersection essential for exploring flows on sections}

The basic elements of the roundabout for counting vehicles are approaches, entry and exit lanes by approaches, sections between adjacent access roads and the radius of the center of the traffic lane at the roundabout [1].

The intersection of the roundabout with three access roads is presented in Figure 1. The three-way roundabout was used to define the model due to the simplicity and possibility of noticing certain dependencies between quantities.

Public city transport at roundabouts can have significant time losses and thus become less competitive compared to a private car [2], [5]. 


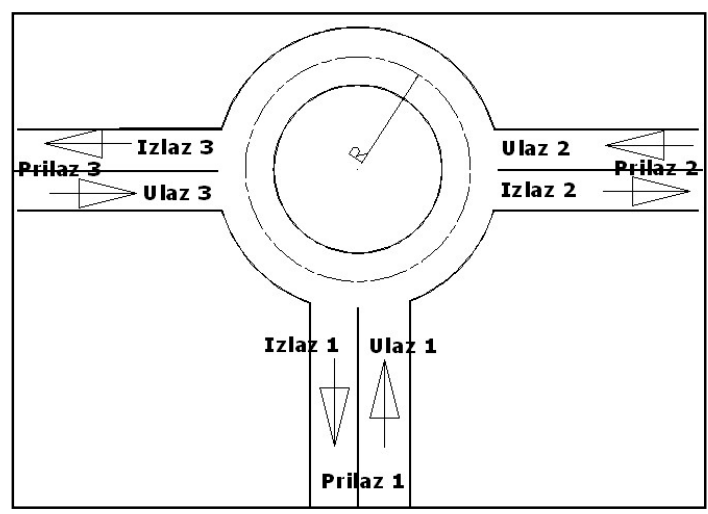

Figure 1. Roundabout with 3 access

The importance of public urban transport in the concept of urban mobility imposes the need to study vehicle flows at roundabouts, of which there are more and more today. Roundabouts are traffic hubs and can be bottlenecks in the traffic flow. Traffic optimization includes the capacity of intersections and even roundabouts [3].

Placing traffic lights at the intersection of a roundabout may not be able to improve the situation if the traffic flow survey does not determine the work plan [8].

\section{Inputs of the MRRF model}

Input values are the basic elements of the intersection and the size determined by counting at the approaches to the intersection. The roundabout, among other things, has $\mathrm{n}$ - approaches and $R(\mathrm{~m})$ - radius of the outer traffic lane [4]. Depending on the average speed of the vehicle in the outer traffic lane and the radius $R$, the time $t c$ that elapses from the entry of the vehicle into the intersection to its exit at the same approach (semicircular turning time) can be determined. Time $t c$ ( $\mathrm{min})$ is a measure of cycle time.

Determining the cycle time $T_{c}$ is important for determining the number of cycles $n_{c}$ during counting. For each cycle (from 0 to $n_{c}$ ) according to the given model, it is possible to determine the flows of vehicles along the sections of the intersection, as well as the flows of vehicles in the directions, and the number of vehicles entering and exiting by approaches.

The duration of the cycle $T_{c}$ can be a period of time (e.g. $1 \mathrm{~min}, 5 \mathrm{~min}, 15 \mathrm{~min}$ and $60 \mathrm{~min}$ ), and is closest to the time of the semicircular rotation of the vehicle $t c$ from any approach. Its mathematical form is depicted in Eq. (1).

$$
t_{c}=\frac{L_{o}}{V_{s}}=\frac{2 \pi R}{\frac{V_{s}}{3,6}}=\frac{2 \cdot 3,6 \cdot \pi \cdot R}{60 \cdot V_{s}}
$$

$$
T_{c}=t_{c}=0,3768 \cdot \frac{R}{V_{s}} \quad(\mathrm{~min})
$$

where is:

Lo (m) - the circumference of the circle of the outer traffic lane

$R(\mathrm{~m})$ - radius of the outer traffic lane

$V_{S}(\mathrm{~km} / \mathrm{h})$ - average speed of the vehicle in the outer traffic lane.

The cycle time $T c$ needs to be re-corrected after calculating and rounding the number of cycles $n_{c}$ to the next integer. For example:

$$
\begin{aligned}
T_{c} & =0,3768 \cdot \frac{R[\mathrm{~m}]}{V_{s}\left[\frac{\mathrm{km}}{h}\right]}=\frac{2 \cdot 3,6 \cdot 3,14 \cdot 30}{60 \cdot 10} \\
T_{c} & =1,13(\mathrm{~min}) .
\end{aligned}
$$

The cycle time depends, among other things, on the driver's behavior [6], [7]. The number of cycles $n_{c}$ during counting is calculated by dividing the recording duration time by the duration of a single cycle as shown in Eq. (3).

$$
n_{c}=\frac{T_{s n}}{T_{c}} ; \quad T_{s n}=t_{k}-t_{p}
$$

where is:

$T_{s n}$ - counting time

$T_{c}$ - cycle time

$t_{p}$ - start of counting (time of entry of the first vehicle)

$t_{k}$ - end of recording (time of exit of the last vehicle).

Finally, the number of cycles is an integer and can be calculated by using Eq. (4).

$$
\frac{T_{s n}}{T_{c}}, n_{c}=I N T\left(\frac{T_{s n}}{T_{c}}\right)
$$

After rounding the number of cycles, it is necessary to recalculate the cycle time. Therefore, we have result shown in Eq. (5).

$$
T_{c}=\frac{T_{s n}}{n_{c}}(\mathrm{~min})
$$

For example:

$$
\begin{aligned}
& t_{p}=00: 00 ; t_{k}=24: 00 ; T_{c}=1,13(\mathrm{~min}) \\
& T_{s n}=24: 00-00: 00=24(\mathrm{~h})=1440(\mathrm{~min}) \\
& n_{c}=\frac{1440}{1,13}=1274,336(\text { cycle }) \\
& n_{c}=\operatorname{INT}\left(\frac{1440}{1,13}\right)=\operatorname{INT}(1274,336)=1274
\end{aligned}
$$

This form can be adopted as follows Eq. (2): 
The final cycle time is:

$$
T_{c}=\frac{T_{s n}}{n_{c}}=\frac{1440}{1274}=1,13029 \approx 1,13(\mathrm{~min})
$$

The numbers determine the numbers of approaches $U_{i}$ and exits $I_{i}$ of vehicles at the intersections. $U_{i}^{k}$ are inclusions in the $i$-th input and $k$-th cycle. $I_{i}^{k}$ are exclusions in the $i$-th output (input) and the $k$-th cycle. The counters count the vehicles entering the intersection regardless of which exit they will be disconnected from the flow, and at the exits they count the vehicles that were disconnected from the flow regardless of which entrance they entered the intersection.

\section{Model MRRF description} in:

The basic application of the MRRF model is reflected

- Determining the flow of vehicles by sections of the intersection, in total and by structure

- Determining the number of vehicles by directions

- Determining the scope of intersection services.

\subsection{Determination of vehicle flow on sections of} roundabout based on vehicle entrances and exits

Based on the previously described input quantities, the deductive method defined a mathematical model for calculating the flow of vehicles per section of the intersection. The following describes the ratios of $U$ vehicle entrances to the intersection, $I$-vehicle exits from the intersection and $q$-vehicle flow per section between the two approaches of the roundabout with 3 approaches. Cycles were observed starting from the zero cycle when there are no vehicles in the intersection and therefore the number of exits at the first approach is $I_{l}{ }^{0}=0$.

Vehicle flows $q_{i}{ }^{0}$ - per section or $q_{i j}{ }^{0}$ - between adjacent approaches in the zero (initial) cycle are shown in Eq (6).

$$
\begin{aligned}
& q_{1}^{0}=q_{12}^{0}=U_{1}^{0} ; I_{1}^{0}=0 \\
& q_{2}^{0}=q_{23}^{0}=q_{12}^{0}+U_{2}^{0}-I_{2}^{0} \\
& q_{3}^{0}=q_{31}^{0}=q_{23}^{0}+U_{3}^{0}-I_{3}^{0}
\end{aligned}
$$

The flow of vehicles on the section of the intersection is equal to the difference of all approaches and exits of vehicles on the approaches to the beginning of the section. One line stretches along the tram route at a distance of 300 to $1000 \mathrm{~m}$ (on the other side of the river).

$$
\begin{aligned}
& q_{1}^{0}=q_{12}^{0}=U_{1}-I_{1} \\
& q_{2}^{0}=q_{23}^{0}=U_{1}^{0}+U_{2}^{0}-I_{2}^{0} \quad\left(I_{1}^{0}=0\right) \\
& q_{3}^{0}=q_{31}^{0}=U_{1}^{0}+U_{2}^{0}+U_{3}^{0}-I_{2}^{0}-I_{3}^{0}
\end{aligned}
$$

We can generally write for the initial cycle the following Eq. (8).

$$
q_{i}^{0}=\sum_{j=1}^{i} U_{j}^{0}-\sum_{j=1}^{i} I_{j}^{0}(i=1 \text { do } n)
$$

where is:

$n$ - number of approaches

$U_{i}^{0}$ - entries (inclusions) in the initial cycle

$I_{i}^{0}$ - exits in the initial cycle

$q_{i j}^{0}$ - flows in the initial cycle

$i$ - the designation of the section or approach at the beginning of the section

$j$ - approach mark at the end of the section.

In the next (first) full cycle, analogous to the previously described, the relations form Eq. (9) are valid.

$$
\begin{aligned}
& q_{1}^{1}=q_{12}^{1}=q_{31}^{0}+U_{1}^{1}-I_{1}^{1} \\
& q_{2}^{1}=q_{23}^{1}=q_{12}^{1}+U_{2}^{1}-I_{2}^{1} \\
& q_{3}^{1}=q_{31}^{1}=q_{23}^{1}+U_{3}^{1}-I_{3}^{1}
\end{aligned}
$$

Therefore, we have the following Eq. (10):

$$
\begin{aligned}
& q_{1}^{1}=q_{12}^{1}=q_{31}^{0}+U_{1}^{1}-I_{1}^{1} \\
& q_{2}^{1}=q_{23}^{1}=q_{31}^{0}+U_{1}^{1}+U_{2}^{1}-I_{1}^{1}-I_{2}^{1} \\
& q_{3}^{1}=q_{31}^{1}=q_{31}^{0}+U_{1}^{1}+U_{2}^{1}+U_{3}^{1}-I_{1}^{1}-I_{2}^{1}-I_{3}^{1}
\end{aligned}
$$

In general, we can write Eq. (11):

$$
\begin{aligned}
& q_{i}^{1}=q_{n}^{0}+\sum_{j=1}^{n} U_{j}^{1}-\sum_{j=1}^{n} I_{j}^{1} \\
& q_{i}^{1}=\sum_{j=1}^{n} U_{j}^{0}-\sum_{j=1}^{n} I_{j}+\sum_{j=1}^{i} U_{j}^{1}-\sum_{j=1}^{i} I_{j}^{1}
\end{aligned}
$$

where is:

$U_{i}^{0}$ - inclusions in the initial cycle

$I_{i}^{0}$ - exclusions in the initial cycle

$q_{i j}^{0}$ - flows in the initial cycle

$U_{i}^{1} ; I_{i}^{1} ; q_{i j}^{1}$ - in the first full cycle

$i=1$ to $n, n$ - number of approaches

$i$ - the designation of the section or approach at the beginning of the section.

$j$ - approach mark at the end of the section.

For the second cycle we can generally write Eq. (12):

$$
\begin{aligned}
& q_{i}^{2}=q_{n}^{1}+\sum_{j=1}^{i} U_{j}^{2}-\sum_{j=1}^{i} I_{j}^{2} \\
& q_{i}^{2}=\sum_{j=1}^{n} U_{j}^{0}-\sum_{j=1}^{n} I_{j}^{0}+\sum_{j=1}^{n} U_{j}^{1}-\sum_{j=1}^{n} I_{j}^{1}+ \\
& +\sum_{j=1}^{i} U_{j}^{2}-\sum_{j=1}^{i} I_{j}^{2} \\
& q_{i}^{2}=\left(\sum_{j=1}^{n} U_{j}^{0}+\sum_{j=1}^{n} U_{j}^{1}\right)-\left(\sum_{j=1}^{n} I_{j}^{0}+\sum_{j=1}^{n} I_{j}^{1}\right)+ \\
& \sum_{j=1}^{i} U_{j}^{2}-\sum_{j=1}^{i} I_{j}^{2}
\end{aligned}
$$


Finally, according to the expressions for the initial, first and second cycles, for any cycle the flow of vehicles on the section of the roundabout is calculated by the Eq. (13):

$$
\begin{aligned}
& q_{i}^{(k)}=\left(\sum_{j=1}^{n} U_{j}^{0}+\sum_{j=1}^{n} U_{j}^{1}+\ldots+\sum_{j=1}^{n} U_{j}^{k-1}\right)- \\
& -\left(\sum_{j=1}^{n} I_{j}^{0}+\sum_{j=1}^{n} I_{j}^{1}+\ldots+\sum_{j=1}^{n} I_{j}^{k-1}\right)+\sum_{j=1}^{i} U_{j}^{(k)}-\sum_{j=1}^{i} I_{j}^{(k)} \\
& q_{i}^{(k)}=\sum_{l=0}^{k-1} \sum_{j=1}^{n} U_{j}^{l}-\sum_{l=0}^{k-1} \sum_{j=1}^{n} I_{j}^{l}+\sum_{j=1}^{i} U_{j}^{(k)}-\sum_{j=1}^{i} I_{j}^{(k)}
\end{aligned}
$$

Parts of this expression have the following meaning expressed in Eq. (14):

$$
\begin{aligned}
& U S^{(k-1)}=\left(\sum_{j=1}^{n} U_{j}^{0}+\sum_{j=1}^{n} U_{j}^{1}+\ldots+\sum_{j=1}^{n} U_{j}^{k-1}\right)=\sum_{l=0}^{k-1} \sum_{j=1}^{n} U_{j}^{l} \\
& I S^{(k-1)}=\left(\sum_{j=1}^{n} I_{j}^{0}+\sum_{j=1}^{n} I_{j}^{1}+\ldots+\sum_{j=1}^{n} I_{j}^{k-1}\right)=\sum_{l=0}^{k-1} \sum_{j=1}^{n} I_{j}^{l}
\end{aligned}
$$

where is:

$U S^{(k)}$ - the sum of all recorded entries on the approaches to the cycle in the appropriate period. $I S^{(k)}$ the sum of all recorded outputs up to the cycle in the appropriate period. In the shorter form for the cycle $(k)$, the flow is calculated according to the Eq. (15):

$$
q_{i}^{(k)}=U S^{(k-1)}-I S^{(k-1)}+\sum_{j=1}^{i} U_{j}^{(k)}-\sum_{j=1}^{i} I_{j}^{(k)}
$$

where is:

$q_{i}^{(k)}$ - flow at the junction of the intersection from the $i$-th to the approach in the $i+1-$ th cycle.

$U_{j}^{(k)} ; I_{j}^{(k)}$ - recorded entrances and exits of vehicles on the $j$-th approach and $k$-th cycle

\subsection{Determining the flow of vehicles on sections of} a roundabout based on the number of vehicles by directions

The analysis of the dependence of the flow on the recorded numbers of vehicles by directions, ie the number of vehicles between the approaches to the intersection (aproaches / exits), was performed by cycles.

In the initial cycle, the number of entries per approach is calculated as the sum of vehicle entries on the observed approach to other approaches in the intersection, and the number of exits as the sum of vehicle exits from other approaches such as shown in Eq. (16).

$$
\begin{array}{ll}
U_{1}^{0}=u_{11}^{0}+u_{12}^{0}+u_{13}^{0} & I_{1}^{0}=0 \\
U_{2}^{0}=u_{21}^{0}+u_{22}^{0}+u_{23}^{0} & I_{2}^{0}=u_{12}^{0} \\
U_{3}^{0}=u_{31}^{0}+u_{32}^{0}+u_{33}^{0} & I_{3}^{0}=u_{13}^{0}+u_{23}^{0}
\end{array}
$$

where is:

$u_{j l}^{0}$ - vehicle entries in $j$-th approach for $l$-th exit to 0 -th cycle

$j, l$ - direction of vehicle movement from $j$-th $\mathrm{u} l$-th

The first cycle is expressed in Eq. (17):

$$
\begin{array}{ll}
U_{1}^{1}=u_{11}^{1}+u_{12}^{1}+u_{13}^{1} & I_{1}^{1}=u_{11}^{0}+u_{21}^{0}+u_{31}^{0} \\
U_{2}^{1}=u_{21}^{1}+u_{22}^{1}+u_{23}^{1} & I_{2}^{1}=u_{12}^{1}+u_{22}^{0}+u_{32}^{0} \\
U_{3}^{1}=u_{31}^{1}+u_{32}^{1}+u_{33}^{1} & I_{3}^{1}=u_{13}^{1}+u_{23}^{1}+u_{33}^{0}
\end{array}
$$

The second cycle is expressed in Eq. (18):

$$
\begin{array}{ll}
U_{1}^{2}=u_{11}^{2}+u_{12}^{2}+u_{13}^{2} & I_{1}^{2}=u_{11}^{1}+u_{21}^{1}+u_{31}^{1} \\
U_{2}^{2}=u_{21}^{2}+u_{22}^{2}+u_{23}^{2} & I_{2}^{2}=u_{12}^{2}+u_{22}^{1}+u_{32}^{1} \\
U_{3}^{2}=u_{31}^{2}+u_{32}^{2}+u_{33}^{2} & I_{3}^{2}=u_{13}^{2}+u_{23}^{2}+u_{33}^{1}
\end{array}
$$

In general, the relationship between the number of vehicles by direction and the vehicle by approaches at the end of the $k$-th cycle is expressed in Eq. (19).

$$
\begin{aligned}
& U_{j}^{k}=\sum_{l=1}^{n} u_{j l}^{k} ; \quad I_{j}^{k}=\sum_{l=j}^{n} u_{l j}^{k-1}+\sum_{l=1}^{j-1} u_{l j}^{k} \\
& \text { for } j=1 \text { it is } \sum_{l=1}^{j-1} u_{l j}^{k}=0
\end{aligned}
$$

where is:

$U_{j}^{k}$ - the number of vehicles entering the driveway kth cycle.

$I_{j}^{k}$ - the number of vehicles exiting the driveway $\mathrm{j} \mathrm{u} \mathrm{k-}$ th cycle.

$u_{j l}^{k}$ - vehicle entrances to $j$-th approach for $l$-th exit in $k$-th cycle.

Expression (19) referring to the full cycle can also be used to calculate the number of entries and exits of vehicles from the intersection to the approach and, $k$-th cycle, or period of time. Therefore, we have the following Eq. (20).

$$
\begin{aligned}
& U P_{i}^{(k)}=\sum_{j=1}^{i} U_{j}^{(k)}=\sum_{l=1}^{i} u_{j l}^{k} \\
& I P_{i}^{(k)}=\sum_{j=1}^{i} I_{j}^{(k)}=\sum_{l=j}^{n} u_{l j}^{k-1}+\sum_{l=1}^{i-1} u_{l i}^{k}
\end{aligned}
$$

where is:

$U P_{i}^{(k)}$ - the sum of all entrances to the entrances in $k$ th cycle to $i$-th access. 
$I P_{i}^{(k)}$ - the sum of all exits at the entrances in $k$-th cycle to $i$-th access.

By including equations (20) in equation (15), the final expression is obtained for calculating the flow of vehicles per section, i.e. after a certain approach.

$$
\begin{aligned}
& q_{i}^{(k)}=U S^{(k-1)}-I S^{(k-1)}+U P_{i}^{(k)}-I P_{i}^{(k)} \\
& q_{i}^{(k)}=\sum_{l=0}^{k} \sum_{j=1}^{n} U_{j}^{k}-\sum_{l=0}^{k} \sum_{j=1}^{n} I_{j}^{k}+\sum_{j=1}^{i} U_{j}^{(k)}-\sum_{j=1}^{i} I_{j}^{(k)} \\
& q_{i}^{(k)}=\sum_{l=0}^{k-1} \sum_{j=1}^{n} \sum_{l=1}^{n} u_{j l}^{l 1}-\sum_{l=0}^{k-1} \sum_{j=1}^{n} \sum_{l=1}^{n} u_{l j}^{l 1}+ \\
& +\sum_{j=1}^{i} \sum_{l=1}^{n} u_{j l}^{k}-\left(\sum_{l=j}^{n} u_{l j}^{k-1}+\sum_{l=1}^{i-1} u_{l i}^{k}\right)
\end{aligned}
$$

By applying expression (21) for the initial and first cycle, we obtain the following equations:

For zero cycle we have the following Eq. (22):

$q_{1}^{0}=u_{11}^{0}+u_{12}^{0}+u_{13}^{0}$

$q_{2}^{0}=q_{1}^{0}+u_{21}^{0}+u_{22}^{0}+u_{23}^{0}-u_{12}^{0}$

$=u_{11}^{0}+u_{12}^{0}+u_{13}^{0}+u_{21}^{0}+u_{22}^{0}+u_{23}^{0}-u_{12}^{0}$

$q_{2}^{0}=u_{11}^{0}+u_{13}^{0}+u_{21}^{0}+u_{22}^{0}+u_{23}^{0}$

$q_{3}^{0}=q_{2}^{0}+u_{31}^{0}+u_{32}^{0}+u_{33}^{0}-u_{13}^{0}-u_{23}^{0}$

$q_{3}^{0}=u_{11}^{0}+u_{21}^{0}+u_{22}^{0}+u_{31}^{0}+u_{32}^{0}+u_{33}^{0}$

The first full cycle we have the following Eq. (23):

$q_{1}^{1}=q_{3}^{0}+\left(u_{11}^{1}+u_{12}^{1}+u_{13}^{1}\right)-\left(u_{11}^{0}+u_{21}^{0}+u_{31}^{0}\right)$

$q_{1}^{1}=u_{22}^{0}+u_{32}^{0}+u_{33}^{0}+u_{11}^{1}+u_{12}^{1}+u_{13}^{1}$

$q_{2}^{1}=q_{1}^{1}+\left(u_{21}^{1}+u_{22}^{1}+u_{23}^{1}\right)-\left(u_{12}^{1}+u_{22}^{0}+u_{32}^{0}\right)$

$q_{2}^{1}=u_{33}^{0}+u_{21}^{1}+u_{22}^{1}+u_{23}^{1}-u_{12}^{1}$

$q_{3}^{1}=q_{2}^{1}+\left(u_{31}^{1}+u_{32}^{1}+u_{33}^{1}\right)-\left(u_{13}^{1}+u_{23}^{0}+u_{33}^{0}\right)$

$q_{3}^{1}=u_{21}^{1}+u_{22}^{1}+u_{31}^{1}+u_{32}^{1}+u_{33}^{1}-u_{12}^{1}-u_{13}^{1}$

The obtained systems of equations can be used to calculate unknown quantities after counting vehicles at the intersection, as well as to establish other relations between the model sizes.

4.3 Determining the number of vehicles by directions based on the number of vehicles at the entrance and exit observed by the approaches to the intersection

The final expression for calculating the number of vehicles by directions, and on the basis of the determined number of vehicles by approaches, based on the conclusions by cycles, cannot be unambiguously defined.
The reason for that is a larger number of unknowns than the number of equations in the system.

The system of equations Eq. (24) applies to the zero cycle:

$$
\begin{array}{ll}
U_{1}^{0}=u_{11}^{0}+u_{12}^{0}+u_{13}^{0} & I_{1}^{0}=0 \\
U_{2}^{0}=u_{21}^{0}+u_{22}^{0}+u_{23}^{0} & I_{2}^{0}=u_{12}^{0} \\
U_{3}^{0}=u_{31}^{0}+u_{32}^{0}+u_{33}^{0} & I_{3}^{0}=u_{13}^{0}+u_{23}^{0}
\end{array}
$$

Vehicle flows per section are depicted in Eq. (25)

$$
\begin{aligned}
& q_{1}^{0}=U_{1}^{0}-I_{1}^{0} \\
& q_{2}^{0}=q_{1}^{0}+U_{2}^{0}-I_{2}^{0}=\left(U_{1}^{0}+U_{2}^{0}\right)-\left(I_{1}^{0}+I_{2}^{0}\right) \\
& q_{3}^{0}=q_{2}^{0}+U_{3}^{0}-I_{3}^{0}= \\
& =\left(U_{1}^{0}+U_{2}^{0}-U_{3}^{1}\right)-\left(I_{1}^{0}+I_{2}^{0}+I_{3}^{0}\right)
\end{aligned}
$$

The known (recorded) parameters are $U_{i}^{0}$ i $I_{i}^{0}$ on the basis of which flows are calculated $q_{i}^{0}$ by intersection sections. The sizes are unknown $u_{j l}^{0}$ vehicle entries in $j$ -th access for $l$-th exit. The number of unknown parameters is 9 , and the number of equations is 6 , so that the system of equations cannot be solved unambiguously. The solution is possible only if the movements of the vehicle from the entrance to the exit from the intersection are recorded.

4.4 Determining the number of vehicles by directions based on the number of vehicles at the entrance and exit observed by the approaches to the intersection

The volume of services $\left(W_{O}\right)$ of the roundabout - load in a certain period of time (cycle $-k$ ) is a function of the number of entrances per approach or the number of exits per approach. Therefore, it can be expressed in Eq. (26).

$$
W_{O}{ }^{(k)}=\sum_{j=1}^{n} U_{j}^{k} \quad \text { ili } \quad W_{O}{ }^{(k)}=\sum_{j=1}^{n} I_{j}^{k}
$$

where is:

$U_{j}^{k}$ - the number of vehicles entering the approach $\mathrm{j}$ in the $k$-th cycle.

$I_{j}^{k}$ - the number of vehicles exiting the approach $\mathrm{j}$ in the $k$-th cycle.

\section{Application of the MRRF model}

The application of the MRRF model is presented on the example of a crossroads with three approaches and on a concrete example of a roundabout in Sarajevo, Ilidža municipality. 
Example 1: Application of MRRF model at roundabout with three approaches

Input data of the MRRF model for the roundabout, according to fig. 1, are given for four cycles, and without the marked start and end time of the cycle, as well as without the exact time of entry and exit of the vehicle on the approaches.

Zero cycle:

$$
u_{i j}{ }^{0}=\left|\begin{array}{ccc}
u_{11}^{0} & u_{12}^{0} & u_{13}^{0} \\
u_{21}^{0} & u_{22}^{0} & u_{23}^{0} \\
u_{31}^{0} & u_{32}^{0} & u_{33}^{0}
\end{array}\right|=\left|\begin{array}{ccc}
0 & 36 & 28 \\
33 & 0 & 20 \\
21 & 24 & 0
\end{array}\right|
$$

Note: Numbers on the diagonal are semicircular turns, and numbers below the diagonal are included in the outputs in the next cycle.

\begin{tabular}{lcc}
\hline \multicolumn{2}{l}{ Entries by cycles } & Cumulative \\
\hline$U_{1}{ }^{0}=$ & 64 & 64 \\
$U_{2}{ }^{0}=$ & 53 & 117 \\
$U_{3}{ }^{0}=$ & 45 & 162 \\
$U S^{0}=$ & 162 & \\
\hline
\end{tabular}

\begin{tabular}{ccc}
\hline \multicolumn{2}{c}{ Exits by cycle } & Cumulative \\
\hline$I_{1}{ }^{0}=$ & 0 & 0 \\
$I_{2}{ }^{0}=$ & 36 & 36 \\
$I_{3}{ }^{0}=$ & 48 & 84 \\
$I^{0}=$ & 84 & \\
\hline
\end{tabular}

First cycle:

$$
u_{i j}{ }^{1}=\left|\begin{array}{ccc}
0 & 26 & 32 \\
35 & 0 & 19 \\
18 & 28 & 0
\end{array}\right|
$$

\begin{tabular}{lcc}
\hline \multicolumn{2}{l}{ Entries by cycles } & Cumulative \\
\hline$U_{1}{ }^{1}=$ & 58 & 58 \\
$U_{2}{ }^{1}=$ & 54 & 112 \\
$U_{3}{ }^{1}=$ & 46 & 158 \\
$U S^{1}=$ & 158 & \\
\hline
\end{tabular}

\begin{tabular}{lcc}
\hline \multicolumn{2}{l}{ Exits by cycles } & Cumulative \\
\hline$I_{1}{ }^{1}=$ & 54 & 54 \\
$I_{2}{ }^{l}=$ & 50 & 104 \\
$I_{3}{ }^{1}=$ & 51 & 155 \\
$I S^{l}=$ & 155 & \\
\hline
\end{tabular}

Second cycle:

$$
u_{i j}^{2}=\left|\begin{array}{ccc}
0 & 45 & 50 \\
50 & 0 & 40 \\
35 & 45 & 0
\end{array}\right|
$$

\begin{tabular}{lcc}
\hline \multicolumn{2}{l}{ Entries by cycles } & Cumulative \\
\hline$U_{1}{ }^{2}=$ & 95 & 95 \\
$U_{2}{ }^{2}=$ & 90 & 185 \\
$U_{3}{ }^{2}=$ & 80 & 265 \\
$U S^{2}=$ & 265 & \\
\hline
\end{tabular}

\begin{tabular}{lcc}
\hline \multicolumn{2}{l}{ Exits by cycles } & Cumulative \\
\hline$I_{1}{ }^{2}=$ & 53 & 53 \\
$I_{2}{ }^{2}=$ & 73 & 126 \\
$I_{3}{ }^{2}=$ & 90 & 216 \\
$I S^{2}=$ & 216 & \\
\hline
\end{tabular}

Third cycle:

$$
u_{i j}^{3}=\left|\begin{array}{ccc}
0 & 62 & 52 \\
48 & 0 & 43 \\
56 & 45 & 0
\end{array}\right|
$$

\begin{tabular}{ccc}
\hline \multicolumn{2}{l}{ Entries by cycles } & Cumulative \\
\hline$U_{1}{ }^{3}=$ & 114 & 114 \\
$U_{2}{ }^{3}=$ & 91 & 205 \\
$U_{3}{ }^{3}=$ & 101 & 306 \\
$U S^{3}=$ & 306 & \\
\hline
\end{tabular}

\begin{tabular}{lcc}
\hline \multicolumn{2}{l}{ Exits by cycles } & Cumulative \\
\hline$I_{1}{ }^{3}=$ & 85 & 85 \\
$I_{2}{ }^{3}=$ & 107 & 192 \\
$I_{3}{ }^{3}=$ & 95 & 287 \\
$I S^{3}=$ & 287 & \\
\hline
\end{tabular}

Fourth cycle:

$$
u_{i j}^{4}=\left|\begin{array}{ccc}
0 & 72 & 82 \\
51 & 0 & 43 \\
49 & 58 & 0
\end{array}\right|
$$

\begin{tabular}{lcc}
\hline \multicolumn{2}{l}{ Entries by cycles } & Cumulative \\
\hline$U_{1}{ }^{4}=$ & 154 & 154 \\
$U_{2}{ }^{4}=$ & 94 & 248 \\
$U_{3}{ }^{4}=$ & 107 & 355 \\
$U S^{4}=$ & 355 & \\
\hline
\end{tabular}

\begin{tabular}{lcc}
\hline & & \\
\hline \multicolumn{2}{l}{ Exits by cycles } & Cumulative \\
\hline$I_{1}{ }^{4}=$ & 104 & 104 \\
$I_{2}{ }^{4}=$ & 117 & 221 \\
$I_{3}{ }^{4}=$ & 125 & 346 \\
$I^{4}=$ & 346 & \\
\hline
\end{tabular}


The sum of inputs and outputs up to the observed moment (cycle) is given in Table 1. The flow of vehicles per section of the intersection is calculated according to (expression 2) is presented in Table 2 and Figure 2.

Table 1. Vehicle entrances and exits up to the observed cycle

\begin{tabular}{ccccccc}
\hline \multirow{2}{*}{$k-$} & \multicolumn{3}{c}{$U S^{(k-l)}+U j^{k}$} & \multicolumn{3}{c}{$I S^{(k-l)}+I j^{k}$} \\
\cline { 2 - 7 } cycle & access & access & access & access & access & access \\
& 1 & 2 & 3 & 1 & 2 & 3 \\
\hline 0 & 64 & 117 & 162 & 0 & 36 & 84 \\
1 & 220 & 274 & 320 & 138 & 188 & 239 \\
2 & 253 & 343 & 423 & 208 & 281 & 371 \\
3 & 379 & 470 & 571 & 301 & 408 & 503 \\
4 & 460 & 554 & 661 & 391 & 508 & 633 \\
\hline
\end{tabular}

Table 2. Vehicle flow per section of intersection

$\mathrm{q}_{\mathrm{i}}=$\begin{tabular}{|c|c|c|c|}
\hline \multirow{2}{*}{$k-$ cycle } & \multicolumn{3}{|c|}{$i$ - section } \\
\cline { 2 - 4 } & 1 to 2 & 2 to 3 & 3 to 1 \\
\hline 0 & 64 & 81 & 78 \\
\hline 1 & 82 & 86 & 81 \\
\hline 2 & 45 & 62 & 52 \\
\hline 3 & 78 & 62 & 68 \\
\hline 4 & 69 & 46 & 28 \\
\hline
\end{tabular}

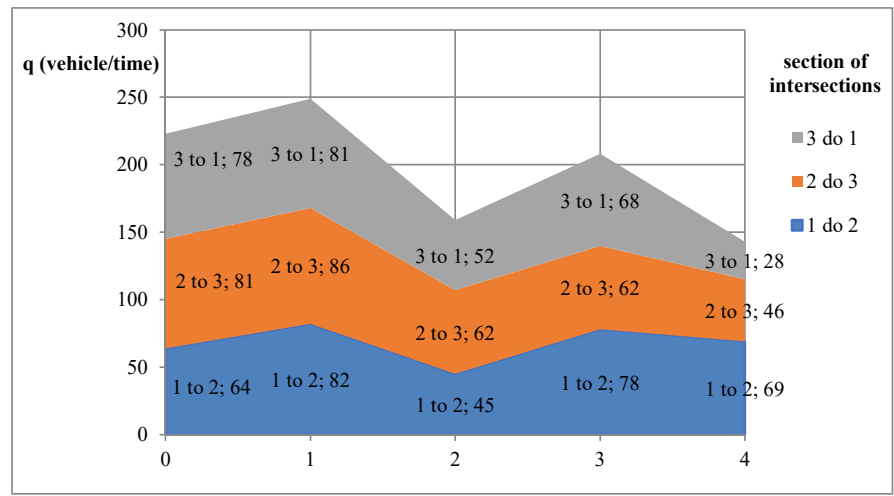

Figure 2. Vehicle flow per intersection section and total for intersection per cycle

Example 2: Application of the MRRF model at the roundabout in Sarajevo (Ilidža)

The roundabout in Ilidža has 6 accesses, 1 of which is only for tram traffic, and one approach is one-way entrance (Figure 3). For trams, there is a traffic light in front of the entrance to the intersection and in the intersection itself,[8] which makes this intersection special.

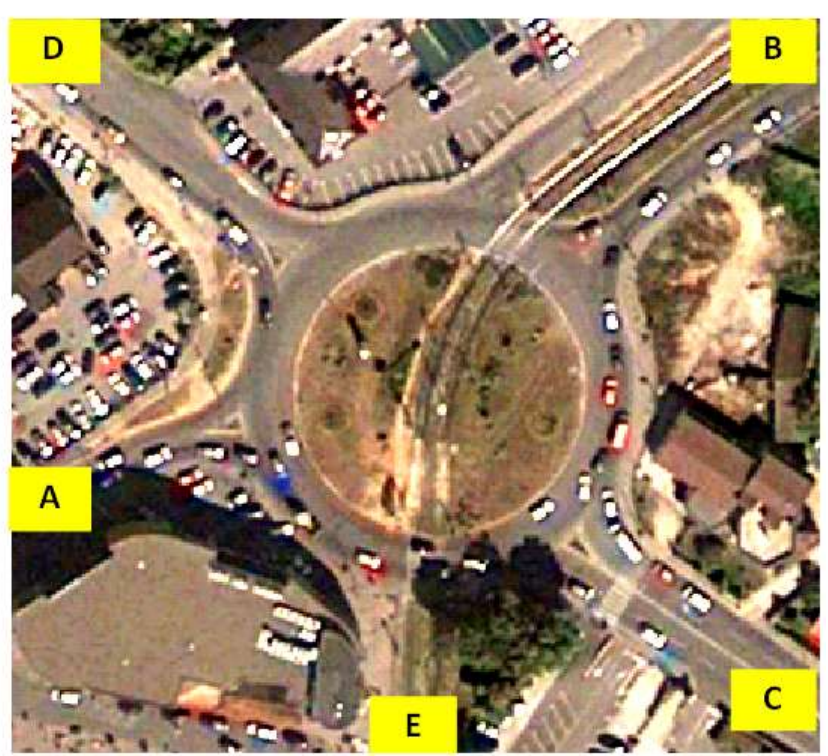

Figure 3 Roundabout, Ilidža

Research of flows at the intersection was conducted with the help of a program developed at the Faculty of Transport and Communications in Sarajevo, Figure 4.

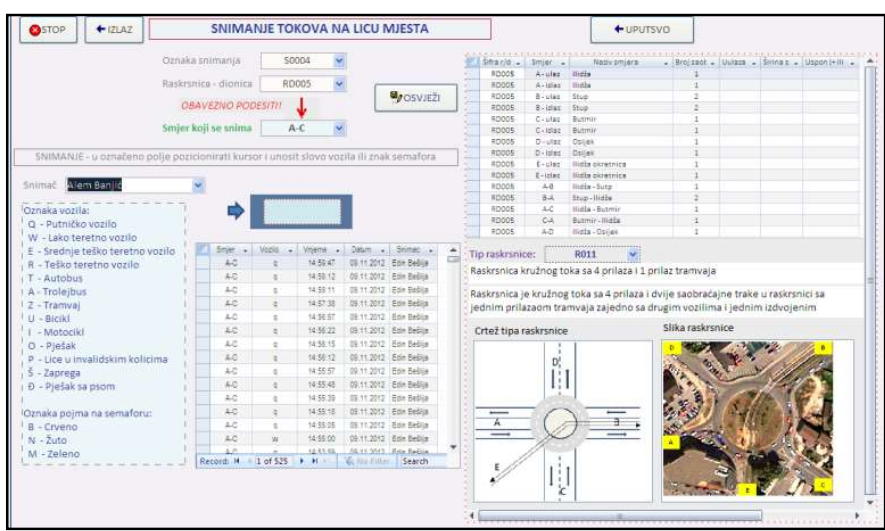

Figure 4. Form of program for research of flows at intersections and road sections

With the help of the mentioned program, the number of vehicles was recorded in such a way that the exact time of entry and exit of vehicles on the approaches was recorded, and by type of vehicle. By selecting an intersection, direction or approach by pressing the appropriate letter on the keyboard, the activity time is entered into the database.

The recording was made on November 9, 2012. from 06:00 to 22:00. In this paper, the period from 12:00 to 15:00 is singled out. The number of vehicle entries by approaches and hours is presented in Table 4 while Table 3 presents vehicle flow per section of intersection in the period 14:00 to 15:00.

The number of vehicles that entered the intersection at all approaches per hour, from 12 to 13 hours 2298, from 13 to 142365 and from 14 to 152629 , represents the scope of service of the intersection by hours. 
The highest flow of vehicles occurs in the period from 14:00 to 15:00 on the section of the intersection between approaches B and D. At approach B, also approximately the largest number of vehicles entered the intersection in the same period.

A traffic light was set up on section A-C and in approach $\mathrm{B}$ at the intersection due to tram traffic that slows down the flow at the intersection.

Table 4. Number of vehicles that entered the intersection by approaches and hours

\begin{tabular}{|c|c|c|c|}
\hline Approaches & 12 do 13 & 13 do 14 & 14 do 15 \\
\hline A & 276 & 383 & 359 \\
\hline B & 975 & 1139 & 1102 \\
\hline C & 662 & 652 & 795 \\
\hline D & 364 & 171 & 350 \\
\hline E & 21 & 20 & 23 \\
\hline Sum & 2298 & 2365 & 2629 \\
\hline
\end{tabular}

Table 5. Vehicle flow per section of intersection in the period 14:00 to $15: 00$

\begin{tabular}{|c|c|c|c|c|}
\hline sections & A-C & C-B & B-D & D-A \\
\hline $\begin{array}{c}q_{i} \\
\text { (vehicle/h) }\end{array}$ & 723 & 664 & 923 & 875 \\
\hline
\end{tabular}

According to the HCM methodology for the analysis of the capacity of roundabouts, it is possible to determine with acceptable accuracy the utilization of access and intersection capacities for single-lane intersections [2].

The capacity (throughput) of the roundabout (C) is represented by the number of vehicles passing the intersection per unit time. It is calculated as the sum of the bandwidth of all approaches $\left(\mathrm{Q}_{\mathrm{Ei}}\right)$ at the roundabout [5].

$$
C=\sum_{i=1}^{n} Q_{E i}(\text { vehicle } / h)
$$

where is:

$Q_{E}$ (vehicle/h) - access bandwidth - input

$n$ - number of approaches

$Q_{E}=f\left(Q_{C}\right.$, dimension $)$

There are several different models for determining the throughput, and for our conditions, with certain adjustments, the Austrian-Swiss model can be used. The access capacity for small and medium roundabouts (linear dependence) is calculated by using Eq. (28).

$$
L=1500-\left[\frac{8}{9}\left(b \cdot M_{K}+a \cdot M_{A}\right)\right](v e h / h)
$$

where is:

$L$ - input throughput [vehicle / h]

$M K$ - load on the roundabout (in the area of the conflict point) [vehicle / h]

$M A$ - exit traffic load [vehicle / h]

$a$ - input geometry factor $b$ - factor of the number of traffic lanes at the intersection

Factor $b$ expresses the influence of the number of traffic lanes on the approach - entrance. The limits of value according to the Austrian-Swiss experience are:

a) with one traffic lane $b=0.90-1.00$

b) with two traffic lanes $b=0.60-0.80$

c) with three traffic lanes $b=0.50-0.60$.

The extent to which the access-saturation is saturated is determined by the degree of load, and is calculated according to the expression

$$
A=\frac{c \cdot M_{E}}{L} 100(\%)
$$

where is:

$A$ - approach load level - input [\%]

$M_{E}$ - input load [train / h]

$L$ - ability to enter [train / $\mathrm{h}$ ]

$c$ - factor of the number of traffic lanes at the entrance.

According to Austrian-Swiss experience, the factor of the number of traffic lanes is:

a) single-track input $c=1.00$

b) two-lane input $c=0.60-0.70$

c) three-lane input $c=0.50$

Table 6 shows the values of the parameters of the roundabout in Ilidža according to the HCM methodology with modification of the factors in accordance with specific conditions. Minor errors are possible due to, in this example, the omission of the tram approach E.

Table 6. Parameters of the intersection in Ilidža in the period 14:00 to $15: 00$ on November 9, 2012.

\begin{tabular}{|l|c|c|c|c|}
\hline \multirow{2}{*}{ Parameter } & \multicolumn{4}{|c|}{ Access } \\
\cline { 2 - 5 } & $A$ & $B$ & $C$ & $D$ \\
\hline $\mathrm{M}_{\mathrm{e}}$ (input load) & 359 & 1102 & 795 & 350 \\
\hline $\mathrm{M}_{\mathrm{A}}$ (output load) & 532 & 521 & 699 & 350 \\
\hline $\mathrm{M}_{\mathrm{K}}$ (load on the roundabout) & 723 & 664 & 923 & 875 \\
\hline a (input geometry factor) & 0,3 & 0,3 & 0,3 & 0,3 \\
\hline $\begin{array}{l}\mathrm{b} \text { (factor of the number of traffic } \\
\text { lanes at the intersection) }\end{array}$ & 0,6 & 0,6 & 0,6 & 0,6 \\
\hline $\begin{array}{l}\mathrm{c} \text { (factor of the number of traffic } \\
\text { lanes at the entrance) }\end{array}$ & 1,0 & 0,65 & 1,0 & 1,0 \\
\hline $\begin{array}{l}\mathrm{L}(\text { ability to enter) } \\
\text { A (\%) (degree of access load - } \\
\text { entrance) }\end{array}$ & 973 & 1007 & 821 & 940 \\
\hline
\end{tabular}

The intersection capacity is $C=3741$ (vehicle/h), and the intersection load is $W_{O}=2606$ (vehicle/h). It can be seen from the previous example that by applying the MRRF method, it is possible to obtain input data for modifying the expression for determining the capacity of the roundabout at the level with several approaches and two or more traffic lanes at the intersection in a simpler way. 


\section{Conclusion}

The described model (MRRF) can be used in determining the flow of vehicles on the sections of the roundabout based on the recorded entrances and exits of the vehicles on the approaches. In addition, by applying the model, it is possible to analyze the effects of individual flows on the capacity of the intersection, and to analyze the number of vehicle movements in the directions of the intersection and their impact on the capacity of the intersection. Vehicle counting is simpler both manually and with the application of new technologies. The model (MRRF) enables results in a longer and shorter period of time, which gives it both static and dynamic characteristics. The presented model can be applied except in intersections of roundabouts in the level and in intersections of other types in the level regardless of the number of approaches.

\section{References}

[1] M. Mehanović, "Planiranje u saobraćaju, prevozu $i$ komunikacijama“, Faculty of Traffic and Communications, University of Sarajevo, 2017.

[2] R. Buehler, J. Pucher, "Demand for public transport in Germany and the USA: an analysis of rider characteristics," Transport Reviews, vol. 32, no. 5, pp. 541-567, 2012.

[3] M. Mehanović, "Mreže u saobraćaju $i$ komunikacijama," Univerzitet u Sarajevu, Fakultet za saobraćaj i komunikacije, Sarajevo, 2015.

[4] Direkcija cesta Federacije BiH, JP Putevi Republike Srpske, "Smjernice za projektiranje, građenje, održavanje $i$ nadzor na putevima u BiH, Sarajevo/Banja Luka," 2005.

[5] M. Mehanović, "Planiranje ponude usluga u gradskom prometu putnika," Faculty of Traffic and Comunication in Sarajevo, University of Sarajevo, 2011.

[6] S. Kaplan, M.A. Guvensan, A.G. Yavuz, Y. Karalurt, "Driver behavior analysis for safe driving: A survey," IEEE Trans. Intell. Transp. Syst, 16, 3017-3032, 2015.

[7] N. Mukai, M. Hayashi, Analysis of Driving Behaviors at Roundabout Intersections by Using Driving Simulator. In: Intelligent Interactive Multimedia Systems and Services, Springer: Berlin/Heidelberg, Germany, 2015, pp. 347-355.

[8] Y. Chimdessa, S. Kassa, L. Obsu (2013). Efficiency of Roundabouts as Compared to Traffic Light Controlled Intersections in Urban Road Networks, "Momona Ethiopian Journal of Science, ” vol. 5, no. 2, pp. 81-100, 2013. 